\title{
Liver anatomy: from Couinaud to the transplantation
}

\author{
Fabrice Duparc $^{1}\left(\mathbb{1} \cdot\right.$ Bruno Grignon $^{2} \cdot$ François Bonnel $^{3} \cdot$ Veronica Macchi $^{4} \oplus \cdot$ Richard Douard $^{5} \oplus$
}

Published online: 31 October 2020

(c) Springer-Verlag France SAS, part of Springer Nature 2020

The last 2020 issue is devoted to the anatomy of the liver. Some anatomical preparations, out of the 250 liver corrosions made by Couinaud and Rapp (1953) that can be seen in the Museum of Anatomy of Montpellier, the oldest Medical University in France, are presented. These studies led to the depiction of the segmentation of the liver and contributed to the great improvement of the anatomical knowledge with a strong clinical and surgical relevance. Three articles show different outer aspects of the liver, related to the grooves and sulcus variations, with emphasizing the importance of these landmarks seen on the hepatic surface during the hepatobiliary surgery.

All the interventional procedures impose an acute preoperative assessment of the hepatic vasculature, and this point sustains the mandatory knowledge of anatomical variations, even rare, and the importance of the medical imaging in a clear viewing of portal branches, hepatic veins and arteries. Besides hepatic surgery, a wide range of liver

Fabrice Duparc

fabrice.duparc@univ-rouen.fr

Bruno Grignon

b.grignon@chu-nancy.fr

François Bonnel

profbonnel@free.fr

Veronica Macchi

veronica.macchi@unipd.it

Richard Douard

richard.douard@clinique-bizet.com

1 Laboratory of Anatomy, Medicine and Pharmacy Faculty, Rouen Normandy University, 22 boulevard Gambetta, 76183 Rouen, France

2 Department of Anatomy, University of Lorraine, Avenue de la Forêt de Haye, 54505 Vandoeuvre-Les-Nancy, France

3 Laboratory of Anatomy, Medicine Faculty, Montpellier University, 2 rue école de medicine, 34000 Montpellier, France

4 Institute of Human Anatomy, Department of Neurosciences, University of Padova, Padua, Italy

5 Clinique Bizet, 23 rue Georges Bizet, 75116 Paris, France interventional radiologic procedures have been developed over the last decades, such as transarterial tumor embolization, chemoembolization, or hepatic radio-embolization, among many others, requiring the same perfect anatomical knowledge.

The correlations between the $3 \mathrm{D}$ representation and the anatomical specimens of Couinaud and Rapp support the incredible contribution of these surgeons-anatomists.

On a more basic point of view, anatomic variations may be of paramount importance for the understanding of pseudolesions of the liver, as is the case with "Sappey's" veins". The "Sappey's veins" are not often considered but can be concerned by the development of a collateral blood flow with cavo-portal anastomosis in cases of superior vena cava obstruction.

More frequently, the anatomical variations can also be seen with the choledochal duct, the gallbladder and cystic duct, even in pediatrics patients.

Finally, a topic of researches remains unsolved: could the hepatic artery be a "non-atherosclerotic" artery?

We wish you a very interesting reading!

Publisher's Note Springer Nature remains neutral with regard to jurisdictional claims in published maps and institutional affiliations. 